\title{
Morbilidad de la zona dadora de injerto óseo autólogo de cresta ilíaca por vía posterior. Análisis de dos técnicas quirúrgicas
}

\author{
Pedro L. Bazán," Jorge D. Cancinos," Álvaro E. Borri," Nicolás Romano Yalour" \\ *Departamento de Columna, Hospital Italiano de La Plata, Buenos Aires, Argentina \\ ** Ortopedia y Traumatología, Sanatorio Ipensa, La Plata, Buenos Aires, Argentina \\ "Servicio de Ortopedia y Traumatología, Hospital Italiano de La Plata, Buenos Aires, Argentina
}

\section{RESUMEN}

Introducción: El injerto óseo autólogo tomado de la cresta ilíaca posterior para artrodesis de columna presenta ventajas: es osteogénico, osteoinductor y osteoconductor, y sus desventajas son ofrecer una cantidad limitada y las complicaciones del sitio dador (8-39\%), como dolor de la zona dadora, lesión neurovascular y fracturas de pelvis. Los objetivos de este estudio fueron comparar la toma de injerto cortico-esponjoso de cresta ilíaca posterior mediante técnica con escoplo gubia frente a la toma con cureta; evaluar las complicaciones intraoperatorias y posoperatorias; cuantificar la cantidad recolectada y graduar el dolor en la zona dadora. Materiales y Métodos: Estudio prospectivo aleatorizado de 34 pacientes consecutivos para artrodesis posterolateral de columna torácica y lumbosacra (26 mujeres y 8 hombres, de entre 15 y 79 años de edad). Se los dividió en dos grupos: grupo 1, cureta y grupo 2, escoplo gubia. Se evaluaron el peso obtenido, el tiempo requerido y las complicaciones. Resultados: Grupo 1: 19 pacientes (14 mujeres y 5 hombres). Se requirieron, en promedio, 9.94 min para obtener 9,26 g. En el primer control, 13 pacientes presentaron un valor $1 ; 5$, un valor 2 y uno, un valor 3 . En el tercer control, 15 tuvieron un puntaje $1 ; 2$, un puntaje 2 y 2 , un puntaje 3 . Grupo 2 : 15 pacientes (12 mujeres y 3 hombres). Se tomaron 11,26 g en 8,6 min. En puntaje de dolor en el primer control fue: 10 pacientes con un valor $1 ; 2$, con un valor 2 y 3 , con un valor 3 , todos tuvieron un puntaje 1 en el tercer control. Conclusiones: La toma de injerto de cresta ilíaca posterior utilizando escoplo gubia es más rápida, recolecta más injerto y provoca menos dolor a los 60 días. Palabras clave: Injerto; autólogo; artrodesis, dolor.

Nivel de Evidencia: II

Autologous donor site morbidity after posterior iliac crest bone harvest. Comparative analysis of two surgical techniques

\section{ABSTRACT}

Introduction: The autologous bone graft harvested from the posterior iliac crest for spinal fusion presents osteogenic, osteoinductive and osteoconductive advantages; however, its disadvantages include a limited amount of available material and an incidence of donor site complications ranging from $8 \%$ to $39 \%$, including donor site pain, neurovascular injury and pelvic fractures. Objectives: To compare the posterior iliac crest corticoancellous harvest using a chisel-gouge approach versus a curette approach; to evaluate intra-operative and post-operative complications; to quantify the harvested bone; to grade donor site pain. Materials and Methods: Prospective randomized study in 34 consecutive patients for posterolateral fusion of the thoracic and lumbosacral spine; 26 women and 8 men, between 15 and 79 years of age. Subjects were divided into two groups. Group 1: curette approach; and Group 2: chiselgouge approach. The evaluation included: the amount of bone harvested, the time required and complications. Results: Group 1: 19 patients, 14 women and 5 men. The procedure lasted an average of $9.94 \mathrm{~min}$, and the harvested material averaged $9.26 \mathrm{~g}$. Denis Pain Scale scores at the first follow-up survey: 13 patients scored 1; 5 scored 2; 1 scored 3. Denis Pain Scale scores at the third follow-up survey: 15 patients scored 1; 2 scored 2; 1 scored 3. Group 2: 15 patients, 12 women and 3 men. The procedure lasted an average of $8.6 \mathrm{~min}$, and the harvested material averaged $9.26 \mathrm{~g}$. Denis Pain Scale scores at their first follow-up: 10 patients scored 1; 2 scored 2; 3 scored 3. At the third follow-up, all patients scored 1. Conclusions: We observed that the posterior iliac crest graft harvested using the chisel-gouge approach is faster, provides more graft and results in less pain at 60 days.

Key words: Graft; autologous; arthrodesis, pain.

Level of Evidence: II

Recibido el 12-3-2017. Aceptado luego de la evaluación el 19-6-2019 • Dr. PEDRO L. BAZÁN • pedroluisbazan@gmail.com

Cómo citar este artículo: Bazán PL, Cancinos JD, Borri ÁE, Romano Yalour N. Morbilidad de la zona dadora de injerto óseo autólogo de cresta ilíaca por vía posterior. Análisis de dos técnicas quirúrgicas. Rev Asoc Argent Ortop Traumatol 2020;85(1):31-38. https://doi.org/10.15417/issn. 1852-7434.2020.85.1.704 


\section{INTRODUCCIÓN}

El injerto autólogo para la artrodesis posterolateral de columna lumbosacra tiene ventajas significativas: es osteogénico, osteoinductor y osteoconductor, pero las desventajas son su cantidad limitada y las complicaciones del sitio dador. ${ }^{1,2} \mathrm{El}$ uso de injerto óseo no estructural, tomado de la cresta ilíaca por vía posterior es bien conocido y su técnica ha sido descrita hace más de 50 años.

Como toda práctica médica no está libre de complicaciones, la incidencia oscila entre el $8 \%$ y el 39\%, ${ }^{2,3}$ y predominan el dolor de la zona dadora, la lesión neurovascular y las fracturas de pelvis. ${ }^{2}$

El dolor en el sitio de toma del injerto se debe a la lesión ocasionada a las terminaciones sensitivas de distintos trayectos nerviosos, nervios cluneales, que pueden resultar dañados por el tamaño de la incisión, como así también por la tracción de los tejidos (Figura 1). ${ }^{1-8}$

Los objetivos de este estudio fueron: 1) comparar la toma de injerto cortico-esponjoso de cresta ilíaca mediante técnica con escoplo gubia, con la toma de injerto esponjoso mediante técnica con cureta, 2) evaluar las complicaciones intraoperatorias y posoperatorias de ambas técnicas, 3) cuantificar la cantidad recolectada buscando diferencias entre ambas y 4) graduar el dolor en la zona dadora.



Figura 1. A. Variación de nervios cluneales y relaciones anatómicas. B. Zona sombreada que muestra la región donde se presenta el dolor asociado a la lesión de nervios cluneales.

\section{MATERIALES Y MÉTODOS}

Se llevó a cabo un estudio prospectivo, a ciego, con decisión de técnica de manera aleatoria, durante 12 meses, para comparar la morbilidad de la zona dadora de cresta ilíaca posterior en 34 pacientes consecutivos (26 mujeres y 8 hombres, rango de edad 15-79 años [promedio 52.4]), que requirieron artrodesis posterolateral de columna torácica y lumbosacra.

Los criterios de inclusión fueron: cirugías primarias para artrodesis por vía posterior, segmento comprendido desde L1 hasta L4 y posibilidad de control telefónico. Se excluyó a los pacientes con antecedentes de trauma o cirugías en ilíacos, escoliosis, tumores, infecciones.

El injerto siempre se tomó por segunda incisión, llevada a cabo no por el cirujano principal y, para su recolección, se utilizaron dos metodologías diferentes: cureta (Figura 2) y escoplo gubia (Figura 3). El uso de la metodología se decidió, en forma aleatoria, mediante un sorteo minutos antes de comenzar la toma. Se confeccionaron 20 cartones con la opción 1 y 20 cartones con la opción 2 . El auxiliar de enfermería retiraba uno al azar. 


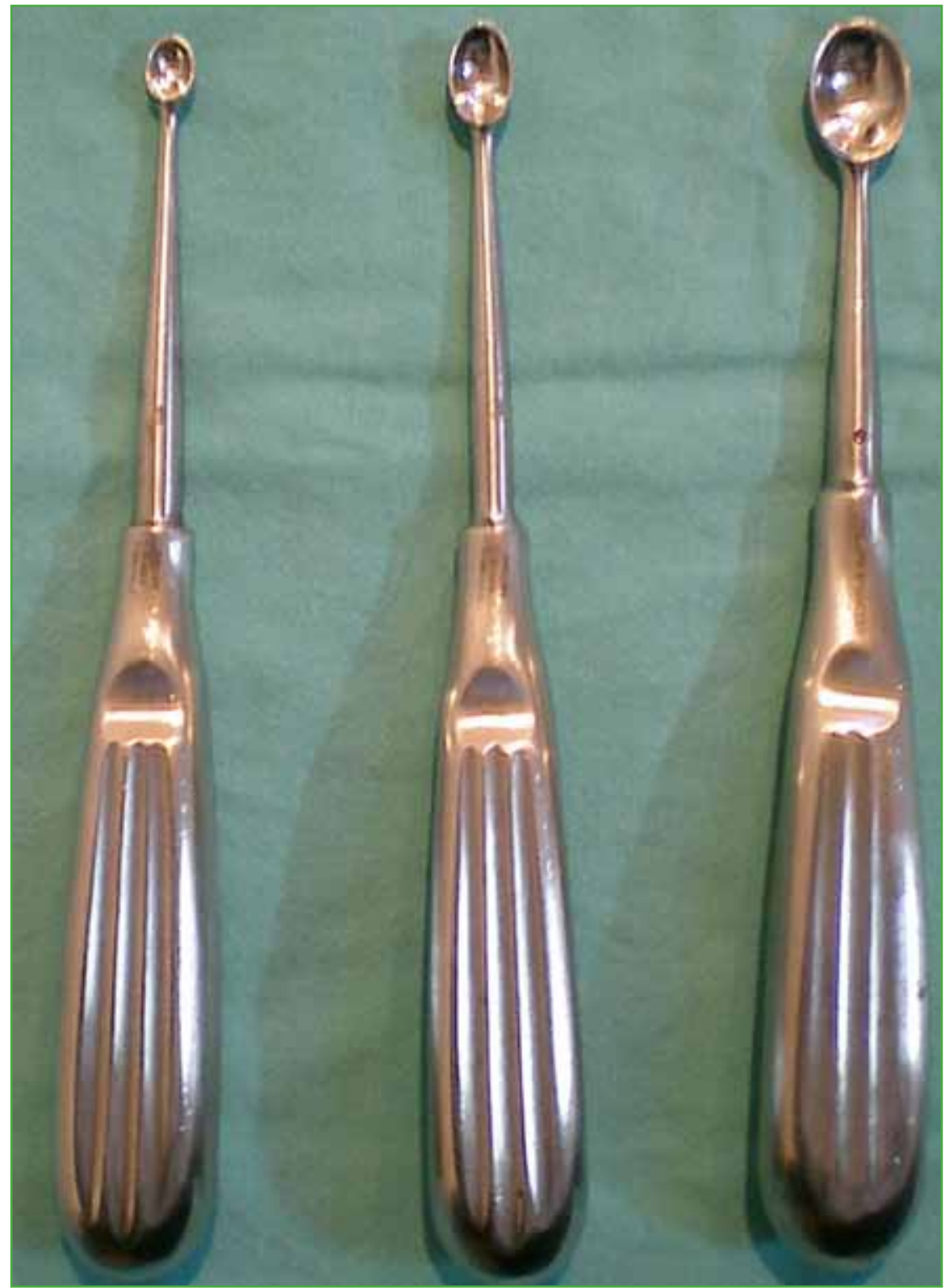

Figura 2. Tipos de curetas utilizadas para la toma de injerto óseo de cresta ilíaca.



Figura 3. Escoplo gubia utilizado para la toma de injerto óseo de cresta ilíaca. 
El material obtenido por toma de cresta ilíaca se pesó en una balanza digital (Figura 4) y separado del obtenido en el campo operatorio.

Se cronometró la duración del procedimiento desde el comienzo del tiempo óseo hasta la obtención completa del material esponjoso. Se detallaron las complicaciones intraquirúrgicas de la técnica.

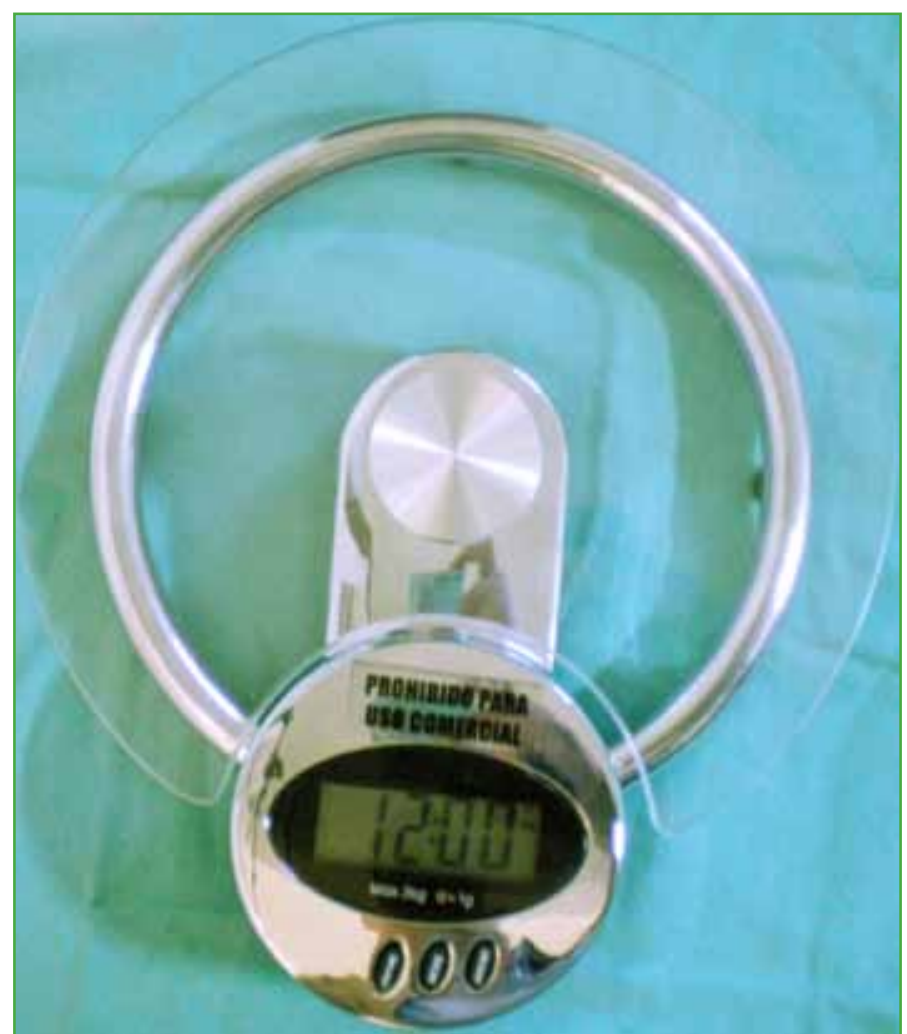

Figura 4. Balanza digital para pesar el injerto óseo tomado de cresta ilíaca y del campo.

Para la evaluación posoperatoria, un empleado administrativo, que desconocía la técnica utilizada al igual que el paciente, realizó una encuesta telefónica a los 15, 30 y 60 días en todos los casos, y a los 90 y 180 días a aquellos que tuvieron dolor en los primeros controles. Se evaluaron el dolor con la escala de Denis (Tabla 1) y las complicaciones en la zona dadora (infección, cicatrización, etc.).

Los resultados se analizaron con el sistema EpiInfo, mediante pruebas de muestras independientes, ji al cuadrado de Pearson.

\section{RESULTADOS}

\section{Grupo 1 (cureta)}

Este grupo estaba conformado por 19 pacientes (14 mujeres y 5 hombres; promedio de edad 52.06).

El tiempo óseo promedio fue de 9.94 min (rango 5-15) y se obtuvo un promedio de 9,26 g (rango 4-16).

Con respecto a la evaluación del dolor mediante la escala de Denis, en el primer control, 13 pacientes tenían un valor 1 ; cinco, un valor 2 y solo uno tenía un valor 3 . Catorce tenían un valor 1 ; cuatro, un valor 2 y uno continuaba con un valor 3 en el segundo control. En el tercer control, 15 pacientes presentaron un valor 1; dos, un valor 2 y otro un valor 3 (Tabla 2). Solo dos pacientes (casos 11 y 12) continuaron el control, porque tenían un valor 2 a los 90 días, de ellos, uno (paciente 12) pasó a un valor 1 a los 180 días. 


\section{Tabla 1. Escala de dolor de Denis}

P1: Sin dolor

P2: Dolor mínimo y ocasional; no requiere medicación

P3: Dolor moderado, medicación ocasional, pero no interrumpe el trabajo o las actividades de la vida diaria

P4: Dolor de moderado a severo, ocasionalmente interrumpe las tareas laborales; cambios significativos de las actividades de la vida diaria

P5: Dolor severo y constante; medicación para dolor crónico

Tabla 2. Dolor en el grupo 1 (cureta)

\begin{tabular}{|c|c|c|c|}
\hline \multirow{2}{*}{ Paciente } & \multicolumn{3}{|c|}{ Dolor } \\
\hline & 1 & 2 & 3 \\
\hline 2 & 2 & 3 & 1 \\
\hline 5 & 1 & 1 & 1 \\
\hline 8 & 2 & 1 & 1 \\
\hline 10 & 1 & 1 & 1 \\
\hline 11 & 2 & 2 & 2 \\
\hline 12 & 3 & 1 & 3 \\
\hline 15 & 1 & 2 & 1 \\
\hline 16 & 2 & 2 & 1 \\
\hline 17 & 1 & 1 & 1 \\
\hline 19 & 1 & 1 & 1 \\
\hline 24 & 1 & 1 & 2 \\
\hline 26 & 1 & 1 & 1 \\
\hline 28 & 1 & 1 & 1 \\
\hline 29 & 2 & 1 & 1 \\
\hline 30 & 1 & 1 & 1 \\
\hline 31 & 1 & 1 & 1 \\
\hline 32 & 1 & 1 & 1 \\
\hline 33 & 1 & 1 & 1 \\
\hline 34 & 1 & 2 & 3 \\
\hline
\end{tabular}

En el primer control, dos pacientes tenían secreción de la herida: uno (paciente 2) serosa y otro (paciente 8) serohemática. No se observó ningún tipo de secreción en los siguientes controles.

El paciente 8 presentó dehiscencia de la herida solo en el primer control.

\section{Grupo 2 (escoplo gubia)}

A este grupo ingresaron 15 pacientes (12 mujeres y 3 hombres; promedio de edad 52.4). El tiempo óseo promedio fue de 8,6 min (rango 3-19) y se obtuvo un promedio de 11,26 g (rango 6-21). 
Con respecto al dolor, según la escala de Denis, en el primer control, 10 pacientes presentaban un valor 1; dos, un valor 2 y solo tres, un valor 3. En el segundo control, 13 pacientes tenían un valor 1, dos, un valor 2 y ninguno, un valor 3. En el tercer control, todos presentaron un valor 1 (Tabla 3).

Tabla 3. Dolor en el grupo 2 (escoplo gubia)

\begin{tabular}{|c|c|c|c|}
\hline \multirow{2}{*}{ Paciente } & \multicolumn{3}{|c|}{ Dolor } \\
\hline & 1 & 2 & 3 \\
\hline 1 & 3 & 1 & 1 \\
\hline 3 & 1 & 2 & 1 \\
\hline 4 & 1 & 1 & 1 \\
\hline 6 & 3 & 1 & 1 \\
\hline 7 & 2 & 1 & 1 \\
\hline 9 & 1 & 1 & 1 \\
\hline 13 & 1 & 1 & 1 \\
\hline 14 & 1 & 1 & 1 \\
\hline 18 & 3 & 2 & 1 \\
\hline 20 & 1 & 1 & 1 \\
\hline 21 & 2 & 1 & 1 \\
\hline 22 & 1 & 1 & 1 \\
\hline 23 & 1 & 1 & 1 \\
\hline 25 & 1 & 1 & 1 \\
\hline 27 & 1 & 1 & 1 \\
\hline
\end{tabular}

En el primer control, un caso (paciente 1) tenía una secreción purulenta de la herida, con cultivo positivo para Staphylococcus aureus. Se le realizó una limpieza quirúrgica y se le administró antibioticoterapia específica. En el segundo control, tenía una secreción serosa y no tuvo secreción en las restantes evaluaciones.

Entre los dos grupos, existen algunas diferencias con respecto al tiempo que demanda cada una de las técnicas: $1.35 \mathrm{~min}(\mathrm{p}>0,05)$ y $2 \mathrm{~g}(\mathrm{p}>0,05)$ en ambas a favor del segundo grupo, estas diferencias no fueron estadísticamente significativas.

Ocurrió algo similar al comparar la evolución del dolor en los tres controles principales, con un promedio de 1,37, 1,32 y 1,32 para el primero, segundo y tercer control del grupo 1; y 1,47, 1,20 y 1 para los mismos controles del grupo 2 ( $\mathrm{p}>0,05)$ (Figura 5).

No se detectaron diferencias con respecto a las otras morbilidades conocidas por la toma de injerto.

\section{DISCUSIÓN}

El injerto autólogo provee una alta osteoinducción y osteoconducción, y tiene una baja inmunogenicidad; por ello, es el elegido por la mayoría de los cirujanos espinales, principalmente en artrodesis largas donde el injerto de campo puede ser escaso. .,2,7 $^{-1}$

El dolor de la zona dadora no es patrimonio de la vía posterior, también es la molestia más frecuente de la toma de injerto por vía anterior, ${ }^{1}$ con menos frecuencia, se puede observar pérdida de la sensibilidad con un pico máximo a los 6 meses. $^{2}$ 




Figura 5. Evolución del dolor posoperatorio en cada uno de los controles, para ambos grupos.

Delawi y cols., ${ }^{4}$ en un estudio sobre la incidencia de dolor en la zona dadora, en pacientes con artrodesis posterior por fracturas vertebrales, hallaron más sintomatología dolorosa en las artrodesis bajas (L3 hacia abajo) que en las altas (L2 hacia arriba). Estos autores concluyen en que el dolor en la zona dadora está sobrevalorado, atribuyen este síntoma a la proximidad de la cirugía lumbar.

Existen varias posibilidades para la toma de injerto óseo de cresta ilíaca por vía posterior. En nuestra práctica, utilizamos dos formas distintas para recolectar el material, con distintos argumentos teóricos para cada una.

La toma mediante el uso de cureta traumatiza menos el periostio y, por esta razón, se cree que causaría menos dolor. En este caso, se requiere descubrir pocos centímetros cuadrados de la cortical de la espina ilíaca posteroexterna y, luego de realizar una ventana con una gubia, se procede a la toma de material exclusivamente esponjoso. Además, puede disminuir la aparición de otras complicaciones conocidas, como lesión vascular, lesión ósea, etc. La desventaja virtual es compactar el injerto por retirar y recolectar menor cantidad. ${ }^{5}$

La toma de injerto óseo de cresta ilíaca en el segundo grupo (mediante escoplo gubia), clásica técnica donde se desinsertan los músculos glúteos y con un escoplo tipo gubia y martillo se toman chips de injerto cortico-esponjoso; las tasas de complicaciones neurovasculares y de lesiones del ilíaco pueden ser más frecuente. Aunque puede traumatizar más el periostio, otorga más injerto. ${ }^{2,4}$

\section{CONCLUSIONES}

Se ha observado que la toma de injerto de cresta ilíaca posterior utilizando un escoplo gubia es más rápida (1,35 min, en promedio), recolecta, en promedio, $2 \mathrm{~g}$ más de injerto óseo y el paciente evoluciona con menos dolor a los 60 días. Estos datos presentan una tendencia hacia la significación.

No hay diferencia con respecto a la presencia de secreción por la herida quirúrgica y su cicatrización entre ambos grupos.

Conflicto de intereses: Los autores no declaran conflictos de intereses. 


\section{BIBLIOGRAFÍA}

1. Silber J, Anderson GD, Daffner SD, Brislin BT, Leland JN, Hilibrand AS, et al. Donor site morbidity after anterior iliac crest bone harvest for single-level anterior cervical discectomy and fusion. Spine (Phila Pa 1976) 2003;28(2):134-9. https://doi.org/10.1097/00007632-200301150-00008

2. Robertson PA, Wray AC. Natural history of posterior iliac crest bone graft donation for spinal surgery. Spine (Phila Pa 1976) 2001;26(13):1473-6. https://doi.org/10.1097/00007632-200107010-00018

3. David R, Folman Y, Pikarsky I, Leitner Y, Catz A, Gepstein R. Harvesting bone graft from the posterior iliac crest by less traumatic, midline approach. J Spinal Disord Tech 2003;(16)1:27-30. https://doi.org/10.1097/00024720200302000-00005

4. Delawi D, Dhert WJ, Castelein, RM, Verbout AJ, Oner FC, The incidence of donor site pain after bone graft harvesting from the posterior iliac crest may be overestimated. Spine (Phila Pa 1976) 2007;32(17):1865-8. https:// doi.org/10.1097/BRS.0b013e318107674e

5. Ebraheim NA, Elgafy H, Xu R. Bone-graft harvesting from iliac and fibular donor sites: techniques and complications. J Am Acad Orthop Surg 2001;9:210- 8. https://doi.org/10.5435/00124635-200105000-00007

6. Ahlmann E, Patzakis M, Roidis N, Shepherd L, Holtom P. Comparison of anterior and posterior iliac crest bone grafts in terms of harvest-site morbidity and functional outcomes. J Bone Joint Surg Am 2002;84(5):716-20. https:// doi.org/10.2106/00004623-200205000-00003

7. Sengupta DK, Truumees E, Patel CK, Kazmierczak C, Hughes B, Elders G, et al. Outcome of local bone versus autogenous iliac crest bone graft in the instrumented posterolateral fusion of the lumbar spine. Spine (Phila Pa 1976) 2006;31:985-91. https://doi.org/10.1097/01.brs.0000215048.51237.3c

8. Gibson S, McLeod I, Wardlaw IM, Urbaniak S. Allograft versus autograft in instrumented posterolateral lumbar spinal fusion. A randomized control trial. Spine (Phila Pa 1976) 2002;27(15):1599-603. https://doi. org/10.1097/00007632-200208010-00002 